\title{
Inverse problem of information diffusion in online social networks
}

\author{
Zvonareva T. ${ }^{*}$, Krivorotko O. ${ }^{1,2}$, Kabanikhin S. ${ }^{1,2}$ \\ ${ }^{1}$ Novosibirsk State University, Novosibirsk, Russia \\ ${ }^{2}$ Institute of Computational Mathematics and Mathematical Geophysics, SB RAS, Novosibirsk, Russia \\ *e-mail: zvonareva-tanyushka@mail.ru
}

Key words: inverse problems, partial differential equations, social processes, diffusive logistic model, optimization, particle swarm optimization

Motivation and Aim: Social network are rapidly progress at the present time: access for informing relatives (Facebook's Safety Check service), publishing data about missing friends (the Google Person Finder service) or promptly informing users of an impending threat and their further actions in case of emergencies (Alerts from Twitter). Such processes can be described by the diffusive logistic mathematical model based on the partial differential equation (PDE) and characterized information dissemination in social networks [1]. The type of information is determined by the coefficients of the mathematical model and the initial conditions of the problem. To control and predict of information in social networks it is necessary to refine the model coefficients and initial data by some additional measurements in fixed time points (the inverse problem) [2]. Methods and Algorithms: One way to solve the inverse problem for PDE is to reduce it to an optimization problem, where the misfit function characterizes the quadratic deviation of the model data from the experimental one. A particle swarm optimization method (PSO) [3] is applied to solve the optimization problem. The algorithm works with a population (called a swarm) of candidate solutions (called particles). It solves a problem by moving these particles around in the search-space according to simple mathematical formulae over the particle's position and velocity.

Results: The reconstructed coefficients and initial data of mathematical models of social networks allow one to refine the information dynamic and give the recommendation for control it.

Acknowledgements: Supported by the Russian Science Foundation (project No. 18-7110044).

\section{References}

1. Wang H., Wang F., Xu K. Modeling information diffusion in online social networks with partial differential equations. arXiv. 2013;1310.0505.

2. Kabanikhin S.I. Definitions and examples of inverse and ill-posed problem. Journal Inverse Ill-Posed Problems. 2008;16(4):317-357.

3. Zhang Y. A comprehensive survey on particle swarm optimization algorithm and its applications. Mathematical Problems Engineering. 2015;931256. 